\title{
A new Toeplitz inversion formula, stability analysis and the value
}

\author{
Yanpeng Zheng ${ }^{\mathrm{a}}$, Zunwei Fub ${ }^{\mathrm{b}}$, Sugoog Shon ${ }^{\mathrm{a}, *}$ \\ ${ }^{a}$ Dept. of Information and Telecommunications Engineering, The University of Suwon, Wau-ri, Bongdam-eup, Hwaseong-si, \\ Gyeonggi-do, 445-743, Korea. \\ ${ }^{b}$ Dept. of Mathematics, The University of Suwon, Wau-ri, Bongdam-eup, Hwaseong-si, Gyeonggi-do, 445-743, Korea.
}

Communicated by $\mathrm{X}$. Qin

\begin{abstract}
In this paper, Toeplitz and Hankel inversion formulae are presented by the idea of skew cyclic displacement. A new Toeplitz inversion formula can be denoted as a sum of products of skew circulant matrices and upper triangular Toeplitz matrices. A new Hankel inversion formula can be denoted as a sum of products of skew left circulant matrices and upper triangular Toeplitz matrices. The stability of their inverse formulae are discussed and their algorithms are given respectively. How the analogue of our formulae lead to a more efficient way to solve the Toeplitz and Hankel linear system of equations are proposed. (C)2017 All rights reserved.
\end{abstract}

Keywords: Toeplitz matrix, skew circulant matrix, inverse, stability, displacement transform.

2010 MSC: 15A09, 15B05.

\section{Introduction}

Toeplitz matrix has become a satisfactory tool in restoration of signals and images [6, 24, 25]. Toeplitz inversion formulae involving circulant matrices have also been presented in $[1,20,21]$. The GohbergSemencul type formula for fingding inverses of Toeplitz and Hankel matrix with skew circulant matrices have not been exploited. Compared with a cyclic convolution algorithm, the skew cyclic convolution algorithm [7, 23] is able to perform filtering in approximately half of computational cost for real signals. In other words, it is able to perform skew-cyclic convolution procedure with about double speed of cyclic one.

Finding inverse of Toeplitz matrix is a hot topic. Heinig and Rost [11] presented an inversion formula for nonsingular Toeplitz matrix. The method gives the desired solution of fundamental equations, where the right-hand side of one of them is a shifted column of the Toeplitz matrix T. The inversion of Toeplitz matrix can be reconstructed by a low number of its columns and the entries of the original Toeplitz matrix. The result was first observed by Trench [27] and reconstructed by Gohberg and Semencul [9] from its first and last columns of $\mathrm{T}^{-1}$, given that the first component in the first column is nonzero. Through three

\footnotetext{
${ }^{*}$ Corresponding author

Email addresses: zhengyanpeng0702@sina.com (Yanpeng Zheng), fuzunwei@lyu.edu.cn (Zunwei Fu), sshon@suwon. ac.kr (Sugoog Shon)
}

doi:10.22436/jnsa.010.03.19 
columns of $\mathrm{T}^{-1}$ to reconstruct the inverse in [4] Labahn and $\mathrm{Ng}[18,26]$ modified this result. The algorithm of Trench for the inversion of Toeplitz matrices was shown with a detailed proof in [30]. Gohberg and Krupnik [8] proposed that if the last component of the first column is nonzero, then $\mathrm{T}^{-1}$ can be recovered from its first and second columns. Lv and Huang [21] presented a new Toeplitz matrix inversion formula in which the inverse can be denoted as a sum of products of circulant matrices and upper triangular Toeplitz matrices. The inverse of an invertible Toeplitz matrix was presented in the form of Toeplitz Bezoutian of two columns in [10]. Labahn and Shalom [19] proposed that formulae for the inverse of layered or striped Toeplitz matrices in terms of solutions of standard equations are observed. Gohberg-Semencul type formulae for inverse of conjugate-Toeplitz and conjugate-Hankel matrix are mentioned in [13, 15]. In [16], Jiang and Wang presented an innovative patterned matrix, RFPL-Toeplitz matrix, is neither the extension of Toeplitz matrix nor its special case. The stability of the algorithms emerging from Toeplitz matrix inversion formulae is considered in [28]. In [22], Naffouti and Baccari gave a characterization of a class of copositive matrices.

In this paper the idea of skew cyclic displacement structure plays a critical role for finding the inverse of Toeplitz matrix, a new Toeplitz inversion formula can be expressed as a sum of products of skew circulant matrices and upper triangular Toeplitz matrices. It will be shown the number of real arithmetic operations is less than known results for solving the Toeplitz and Hankel linear system of equations.

\section{Toeplitz and Hankel inversion formula}

Lemma 2.1. Let $\mathrm{T}=\left(\mathrm{a}_{\mathrm{i}-\mathrm{j}}\right)_{i, j=1}^{\mathrm{n}}$ be an $\mathrm{n} \times \mathrm{n}$ Toeplitz matrix, then it satisfies the formula

$$
\Xi \mathrm{T}-\mathrm{T} \Xi=v e_{\mathrm{n}}^{\mathrm{T}}-e_{1} v^{\mathrm{T}} \mathrm{J}
$$

where

$$
\begin{aligned}
& \Xi=\left(\begin{array}{cccc}
0 & & & -1 \\
1 & \ddots & & \\
& \ddots & \ddots & \\
& & 1 & 0
\end{array}\right), \quad J=\left(\begin{array}{llll} 
& & & \\
& & 1 & \\
& . . & & \\
1 & & &
\end{array}\right) \\
& e_{1}=\left(\begin{array}{c}
1 \\
0 \\
\vdots \\
0
\end{array}\right), \quad e_{n}=\left(\begin{array}{c}
0 \\
\vdots \\
0 \\
1
\end{array}\right), \quad v=\left(\begin{array}{c}
0 \\
a_{1-n}+a_{1} \\
\vdots \\
a_{-2}+a_{n-2} \\
a_{-1}+a_{n-1}
\end{array}\right)
\end{aligned}
$$

Lemma 2.2. Let $\mathrm{H}=\left(\mathrm{b}_{i+j}\right)_{i, j=0}^{n-1}$ be an $\mathrm{n} \times \mathrm{n}$ Hankel matrix, it satisfies

$$
\Xi H-H \Xi^{\top}=\hat{v} e_{1}^{\top}-e_{1} \hat{v}^{\top},
$$

where $\Xi$ and $\mathrm{e}_{1}$ are given in Lemma 2.1 and

$$
\hat{v}=\left(\begin{array}{c}
0 \\
b_{0}+b_{n} \\
b_{1}+b_{n+1} \\
\vdots \\
b_{n-2}+b_{2 n-2}
\end{array}\right)
$$


Theorem 2.3. Let $\mathrm{T}=\left(\mathrm{a}_{\mathrm{i}-\mathrm{j}}\right)_{\mathrm{i}, \mathrm{j}=1}^{\mathrm{n}}$ be a Toeplitz matrix. If each of the systems of equations $\mathrm{T} x=v, \mathrm{Ty}=\mathrm{e}_{1}$ are solvable, $x=\left(\begin{array}{llll}x_{1} & x_{2} & \cdots & x_{n}\end{array}\right)^{\top}, y=\left(\begin{array}{llll}y_{1} & y_{2} & \cdots & y_{n}\end{array}\right)^{\top}$, then $T$ is invertible and

$$
\mathrm{T}^{-1}=\mathrm{S}_{1} \mathrm{U}_{1}+\mathrm{S}_{2} \mathrm{U}_{2}
$$

where

$$
\begin{aligned}
& \mathrm{S}_{1}=\left(\begin{array}{cccc}
\mathrm{y}_{1} & -\mathrm{y}_{\mathrm{n}} & \cdots & -\mathrm{y}_{2} \\
\mathrm{y}_{2} & \mathrm{y}_{1} & \ddots & \vdots \\
\vdots & \ddots & \ddots & -\mathrm{y}_{\mathrm{n}} \\
\mathrm{y}_{\mathrm{n}} & \cdots & \mathrm{y}_{2} & \mathrm{y}_{1}
\end{array}\right), \quad \mathrm{U}_{1}=\left(\begin{array}{cccc}
1 & -x_{\mathrm{n}} & \cdots & x_{2} \\
& 1 & \ddots & \vdots \\
& & \ddots & -x_{n} \\
& & & 1
\end{array}\right) \\
& \mathrm{S}_{2}=\left(\begin{array}{cccc}
\mathrm{x}_{1} & -\mathrm{x}_{\mathrm{n}} & \cdots & -\mathrm{x}_{2} \\
\mathrm{x}_{2} & \mathrm{x}_{1} & \ddots & \vdots \\
\vdots & \ddots & \ddots & -\mathrm{x}_{\mathrm{n}} \\
\mathrm{x}_{\mathrm{n}} & \cdots & \mathrm{x}_{2} & \mathrm{x}_{1}
\end{array}\right), \quad \mathrm{U}_{2}=\left(\begin{array}{cccc}
0 & \mathrm{y}_{\mathrm{n}} & \cdots & \mathrm{y}_{2} \\
& 0 & \ddots & \vdots \\
& & \ddots & \mathrm{y}_{\mathrm{n}} \\
& & & 0
\end{array}\right)
\end{aligned}
$$

and $S_{1}, S_{2}$ are both skew circulant matrices $[14,17,29]$.

Proof. From Lemma 2.1 and $\mathrm{T} x=v, \mathrm{Ty}=e_{1}$, we have

$$
\Xi \mathrm{T}=\mathrm{T} \Xi+v e_{\mathrm{n}}^{\top}-e_{1} v^{\top} \mathrm{J}=\mathrm{T}\left(\Xi+x e_{\mathrm{n}}^{\top}-\mathrm{y} v^{\top} \mathrm{J}\right)
$$

Then

$$
\begin{aligned}
\Xi^{i} \mathrm{~T} & =\Xi^{i-1} \mathrm{~T}\left(\Xi+x e_{n}^{\top}-y v^{\top} J\right) \\
& =\mathrm{T}\left(\Xi+x e_{n}^{\top}-y v^{\top} J\right)^{i} .
\end{aligned}
$$

Therefore,

$$
\Xi^{i} e_{1}=\Xi^{i} T y=T\left(\Xi+x e_{n}^{T}-y v^{T} J\right)^{i} y
$$

Let

$$
t_{i}=\left(\Xi+x e_{n}^{\top}-y v^{\top} J\right)^{i-1} y, \quad \text { and } \hat{T}=\left(\begin{array}{llll}
t_{1} & t_{2} & \cdots & t_{n}
\end{array}\right) .
$$

Then

$$
\begin{aligned}
& \mathrm{T} t_{i}=\mathrm{T}\left(\Xi+x e_{\mathrm{n}}^{\top}-y v^{\top} J\right)^{i-1} y=\Xi^{i-1} e_{1}=e_{i} \\
& \mathrm{~T} \hat{\top}=\mathrm{T}\left(\begin{array}{llll}
t_{1} & t_{2} & \cdots & t_{n}
\end{array}\right)=\left(\begin{array}{llll}
e_{1} & e_{2} & \cdots & e_{n}
\end{array}\right)=I_{n} .
\end{aligned}
$$

So matrix $\mathrm{T}$ is invertible and $\mathrm{T}^{-1}$ is $\hat{\mathrm{T}}$.

It is easy to get

$$
\begin{aligned}
& t_{1}=y, \quad t_{i}=\left(\Xi+x e_{n}^{\top}-y v^{\top} J\right) t_{i-1}, \quad(i=1,2, \cdots, n), \\
& t_{i}=T^{-1} e_{i}, \quad J e_{i}=e_{n-i+1}, \quad J T J=T^{\top}, \quad J J=I, \quad J^{\top}=J .
\end{aligned}
$$

Then, for $i>1$,

$$
\begin{aligned}
t_{i} & =\Xi t_{i-1}+x e_{n}^{\top} t_{i-1}-y v^{\top} J t_{i-1} \\
& =\Xi t_{i-1}+x e_{n}^{\top} T^{-1} e_{i-1}-y v^{\top} J T^{-1} e_{i-1} \\
& =\Xi t_{i-1}+x e_{n}^{\top} J J T^{-1} J e_{n-i+2}-y v^{\top} T^{-\top} J e_{i-1}
\end{aligned}
$$




$$
\begin{aligned}
& =\Xi t_{i-1}+x e_{1}^{\top} T^{-\top} e_{n-i+2}-y v^{\top} T^{-\top} e_{n-i+2} \\
& =\Xi t_{i-1}+x y^{\top} e_{n-i+2}-y x^{\top} e_{n-i+2} \\
& =\Xi t_{i-1}+y_{n-i+2} x-x_{n-i+2} y
\end{aligned}
$$

According to $t_{1}=y$ and equation (2.2), we have

$$
\begin{aligned}
\mathrm{T}^{-1}= & \left(\begin{array}{cccc}
\mathrm{t}_{1} & \mathrm{t}_{2} & \cdots & \mathrm{t}_{\mathrm{n}}
\end{array}\right) \\
= & \left(\begin{array}{cccc}
\mathrm{y}_{1} & -\mathrm{y}_{\mathrm{n}} & \cdots & -\mathrm{y}_{2} \\
\mathrm{y}_{2} & \mathrm{y}_{1} & \ddots & \vdots \\
\vdots & \ddots & \ddots & -\mathrm{y}_{\mathrm{n}} \\
y_{\mathrm{n}} & \cdots & y_{1} & \mathrm{y}_{1}
\end{array}\right)\left(\begin{array}{cccc}
1 & -x_{\mathrm{n}} & \cdots & x_{2} \\
& 1 & \ddots & \vdots \\
& & \ddots & -x_{n} \\
& & & 1
\end{array}\right) \\
& +\left(\begin{array}{cccc}
x_{1} & -x_{n} & \cdots & -x_{2} \\
x_{2} & x_{1} & \ddots & \vdots \\
\vdots & \ddots & \ddots & -x_{n} \\
x_{n} & \cdots & x_{1} & x_{1}
\end{array}\right)\left(\begin{array}{cccc}
0 & y_{n} & \cdots & y_{2} \\
& 0 & \ddots & \vdots \\
& & \ddots & y_{n} \\
& & & 0
\end{array}\right) .
\end{aligned}
$$

Theorem 2.4. Let $\mathrm{H}=\left(\mathrm{b}_{i+j}\right)_{i, j=0}^{n-1}$ be a Hankel matrix. If each of the systems of equations $\mathrm{Hu}=\hat{v}, \mathrm{~Hz}=\mathrm{e}_{1}$ are solvable, $u=\left(\begin{array}{llll}u_{1} & u_{2} & \cdots & u_{\mathfrak{n}}\end{array}\right)^{\top}, z=\left(\begin{array}{llll}z_{1} & z_{2} & \cdots & z_{\mathfrak{n}}\end{array}\right)^{\top}$, then $\mathrm{H}$ is invertible and

$$
\mathrm{H}^{-1}=\mathrm{L}_{1} \Pi_{1}+\mathrm{L}_{2} \Pi_{2}
$$

where

$$
\begin{aligned}
& \mathrm{L}_{1}=\left(\begin{array}{ccccc}
z_{1} & z_{2} & z_{3} & \cdots & z_{\mathrm{n}} \\
z_{2} & z_{3} & . & z_{\mathrm{n}} & -z_{1} \\
z_{3} & . & . & -z_{1} & -z_{2} \\
\vdots & . \cdot & . & . \cdot & \vdots \\
z_{\mathrm{n}} & -z_{1} & -z_{2} & \cdots & -z_{\mathrm{n}-1}
\end{array}\right), \quad \Pi_{1}=\left(\begin{array}{cccc}
1 & -\mathrm{u}_{1} & \cdots & \mathrm{u}_{\mathrm{n}-1} \\
& 1 & \ddots & \vdots \\
& & \ddots & -\mathrm{u}_{1} \\
& & & 1
\end{array}\right) \\
& \mathrm{L}_{2}=\left(\begin{array}{ccccc}
\mathrm{u}_{1} & \mathrm{u}_{2} & \mathrm{u}_{3} & \cdots & \mathrm{u}_{\mathfrak{n}} \\
\mathrm{u}_{2} & \mathrm{u}_{3} & . \cdot & \mathrm{u}_{\mathrm{n}} & -\mathrm{u}_{1} \\
\mathrm{u}_{3} & . \cdot & . \cdot & -\mathrm{u}_{1} & -\mathrm{u}_{2} \\
\vdots & . \cdot & . \cdot & . \cdot & \vdots \\
\mathrm{u}_{\mathrm{n}} & -\mathrm{u}_{1} & -\mathrm{u}_{2} & \cdots & -\mathrm{u}_{\mathrm{n}-1}
\end{array}\right), \quad \Pi_{2}=\left(\begin{array}{cccc}
0 & z_{1} & \cdots & z_{n-1} \\
& 0 & \ddots & \vdots \\
& & \ddots & z_{1} \\
& & 0
\end{array}\right)
\end{aligned}
$$

and $\mathrm{L}_{1}, \mathrm{~L}_{2}$ are both skew left circulant matrices $[14,17,29]$.

Proof. The proof is similar to that of Theorem 2.3.

\section{Stability analysis}

An algorithm is called forward stable, if all well conditioned problems, the computed solution $\tilde{\chi}$ is closed to the true solution $x$ that means the related error $\frac{\|x-\tilde{x}\|_{2}}{\|x\|_{2}}$ is minimal. Round-off errors occur in the matrix computation. Let $A, B \in C^{n \times n}$ and $\lambda \in C$. If we neglect the $O\left(\epsilon^{2}\right)$ terms, then for any floating-point arithmetic with machine precision $\epsilon$ we have (cf. [31])

$$
f l(\lambda A)=\lambda A+E, \quad\|E\|_{F} \leqslant \epsilon|\lambda|\|A\|_{F} \leqslant \epsilon \sqrt{n}|\lambda|\|A\|_{2},
$$




$$
\begin{aligned}
f l(A+B) & =A+B+E, & \|E\|_{F} \leqslant \epsilon\|A+B\|_{F} \leqslant \epsilon \sqrt{n}\|A+B\|_{2} \\
f l(A B) & =A B+E, & \|E\|_{F} \leqslant \epsilon n\|A\|_{F}\|B\|_{F} .
\end{aligned}
$$

According to the floating-point arithmetic, we have the following theorem.

Theorem 3.1. Let $\mathrm{T}$ be a nonsingular Toeplitz matrix and well conditioned, then the formula provided in Theorem 2.3 is forward stable.

Proof. Assume the solutions $\tilde{x}, \tilde{y}$ are obtained from Theorem 2.3, which are perturbed by the normwise relative errors bounded by $\tilde{\epsilon}$,

$$
\|\tilde{x}\|_{2} \leqslant\|x\|_{2}(1+\tilde{\epsilon}), \quad\|\tilde{y}\|_{2} \leqslant\|y\|_{2}(1+\tilde{\epsilon}) .
$$

Therefore,

$$
\begin{array}{ll}
\left\|S_{1}\right\|_{F} \leqslant \sqrt{n}\|y\|_{2}, & \left\|S_{2}\right\|_{F} \leqslant \sqrt{n}\|x\|_{2}, \\
\left\|U_{1}\right\|_{F} \leqslant \sqrt{n}+\sqrt{1+\|x\|_{2}^{2}}, & \left\|U_{2}\right\|_{F} \leqslant \sqrt{n}\|y\|_{2} .
\end{array}
$$

Using the perturbed solutions $\tilde{x}, \tilde{y}$, the inversion formula in Theorem 2.3 is

$$
\begin{aligned}
\tilde{\mathrm{T}}^{-1} & =\mathrm{fl}\left(\tilde{\mathrm{S}_{1}} \tilde{\mathrm{U}}_{1}+\tilde{\mathrm{S}_{2}} \tilde{\mathrm{U}}_{2}\right) \\
& =\mathrm{fl}\left[\left(\mathrm{S}_{1}+\Delta \mathrm{S}_{1}\right)\left(\mathrm{U}_{1}+\Delta \mathrm{U}_{1}\right)+\left(\mathrm{S}_{2}+\Delta \mathrm{S}_{2}\right)\left(\mathrm{U}_{2}+\Delta \mathrm{U}_{2}\right)\right] \\
& =\mathrm{T}^{-1}+\Delta \mathrm{S}_{1} \mathrm{U}_{1}+\mathrm{S}_{1} \Delta \mathrm{U}_{1}+\Delta \mathrm{S}_{2} \mathrm{U}_{2}+\mathrm{S}_{2} \Delta \mathrm{U}_{2}+\mathrm{E}+\mathrm{F},
\end{aligned}
$$

here, $\mathrm{E}$ contains the error from computing the matrix products and $\mathrm{F}$ is the matrix containing the error which results from subtracting the matrices. For the error matrices $\Delta \mathrm{S}_{1}, \Delta \mathrm{U}_{1}, \Delta \mathrm{S}_{2}$ and $\Delta \mathrm{U}_{2}$, we have

$$
\begin{aligned}
& \left\|\Delta S_{1}\right\|_{F} \leqslant \tilde{\epsilon}\left\|S_{1}\right\|_{F} \leqslant \tilde{\epsilon} \sqrt{n}\|y\|_{2}, \\
& \left\|\Delta S_{2}\right\|_{F} \leqslant \tilde{\epsilon}\left\|S_{2}\right\|_{F} \leqslant \tilde{e} \sqrt{n}\|x\|_{2}, \\
& \left\|\Delta U_{1}\right\|_{F} \leqslant \tilde{\epsilon}\left\|U_{1}\right\|_{F} \leqslant \tilde{\epsilon}\left(\sqrt{n}+\sqrt{1+\|x\|_{2}^{2}}\right), \\
& \left\|\Delta U_{2}\right\|_{F} \leqslant \tilde{\epsilon}\left\|U_{2}\right\|_{F} \leqslant \tilde{\epsilon} \sqrt{n}\|y\|_{2} .
\end{aligned}
$$

It follows that

$$
\begin{aligned}
\|E\|_{2} & \leqslant\|E\|_{F} \\
& \leqslant \epsilon n\left(\left\|S_{1}\right\|_{F}\left\|U_{1}\right\|_{F}+\left\|S_{2}\right\|_{F}\left\|U_{2}\right\|_{F}\right) \\
& \leqslant \epsilon n^{2}\|y\|_{2}\left(\sqrt{1+\|x\|_{2}}+\|x\|_{2}\right) \\
& \leqslant \epsilon n^{2}\|y\|_{2}\left(1+2\|x\|_{2}\right), \\
\|v\|_{2} & \leqslant \epsilon \sqrt{n}\left\|T^{-1}\right\|_{2} .
\end{aligned}
$$

From above,

$$
\left\|\tilde{T}^{-1}-T^{-1}\right\|_{2} \leqslant n(2 \tilde{\epsilon}+n \epsilon)\|y\|_{2}\left(1+2\|x\|_{2}\right)+\epsilon \sqrt{n}\left\|T^{-1}\right\|_{2} .
$$

As $T x=v, T y=e_{1}$, then

$$
\|x\|_{2} \leqslant\left\|T^{-1}\right\|_{2}\|v\|_{2}, \quad\|y\|_{2} \leqslant\left\|T^{-1}\right\|_{2}
$$

Thus, the related error is

$$
\frac{\left\|\tilde{T}^{-1}-T^{-1}\right\|_{2}}{\left\|T^{-1}\right\|_{2}} \leqslant n(2 \tilde{\epsilon}+n \epsilon)\left(1+2\left\|T^{-1}\right\|_{2}\|v\|_{2}\right)+\epsilon \sqrt{n} .
$$

As $\mathrm{T}$ is well conditioned, thus, $\left\|\mathrm{T}^{-1}\right\|_{2}$ is finite. Obviously, $\|v\|_{2}$ is finite. Therefore, the formula presented in Theorem 2.3 is forward stable. 
Through the same method, the following theorem can be obtained.

Theorem 3.2. Let $\mathrm{H}$ be an $\mathrm{n} \times \mathrm{n}$ Hankel matrix and well conditioned, then the formula presented in Theorem 2.4 is forward stable.

\section{Two algorithms on finding $\mathrm{T}^{-1}$ and $\mathrm{H}^{-1}$}

In this section, two algorithms on finding $\mathrm{T}^{-1}$ and $\mathrm{H}^{-1}$ are given.

Algorithm 4.1. By use Theorem 2.3, we proceed with

Step 1. Compute $v=\left(\begin{array}{lllll}0 & a_{1-n}+a_{1} & a_{2-n}+a_{2} & \cdots & a_{-1}+a_{n-1}\end{array}\right)^{\top}$.

Step 2. Compute $x=\left(\begin{array}{lllll}x_{1} & x_{2} & \ldots & x_{n}\end{array}\right)^{\top}$ and $y=\left(\begin{array}{lllll}y_{1} & y_{2} & \ldots & y_{n}\end{array}\right)^{\top}$ by solving the systems of equations

$$
\mathrm{Tx}=v \text {, and } \mathrm{Ty}=e_{1} \text {. }
$$

Step 3. Compute $\mathrm{T}^{-1}$ via formula (2.1).

Algorithm 4.2. By use Theorem 2.4, we proceed with

Step 1. Compute $\hat{v}=\left(\begin{array}{lllll}0 & b_{0}+b_{n} & b_{1}+b_{n+1} & \cdots & b_{n-2}+b_{2 n-2}\end{array}\right)^{\top}$.

Step 2. Compute $u=\left(\begin{array}{llll}u_{1} & u_{2} & \cdots & u_{n}\end{array}\right)^{\top}$ and $z=\left(\begin{array}{llll}z_{1} & z_{2} & \cdots & z_{n}\end{array}\right)^{\top}$ by solving the systems of equations

$$
\mathrm{Hu}=\hat{v} \text { and } \mathrm{Hz}=\mathrm{e}_{1} .
$$

Step 3. Compute $\mathrm{H}^{-1}$ via formula (2.3).

\section{Numerical example}

In this section we give two examples to demonstrate our main result.

Example 5.1. $T$ is a $4 \times 4$ Toeplitz matrix

$$
\mathrm{T}=\left(\begin{array}{llll}
0 & 1 & 0 & 0 \\
1 & 0 & 1 & 0 \\
0 & 1 & 0 & 1 \\
0 & 0 & 1 & 0
\end{array}\right) .
$$

It is obvious that $\mathrm{T}$ is invertible. By Algorithm 4.1, we have

Step 1. Compute $v$ by Lemma 2.1:

$$
v=\left(\begin{array}{l}
0 \\
1 \\
0 \\
1
\end{array}\right) .
$$

Step 2. Compute $x, y$ by the systems of equations $T x=v, T y=e_{1}$ :

$$
x=\left(\begin{array}{l}
0 \\
0 \\
1 \\
0
\end{array}\right), y=\left(\begin{array}{c}
0 \\
1 \\
0 \\
-1
\end{array}\right)
$$


Step 3. Compute $\mathrm{T}^{-1}$ by using the equation (2.1):

$$
\begin{aligned}
\mathrm{T}^{-1} & =\left(\begin{array}{cccc}
0 & 1 & 0 & -1 \\
1 & 0 & 1 & 0 \\
0 & 1 & 0 & 1 \\
-1 & 0 & 1 & 0
\end{array}\right)\left(\begin{array}{cccc}
1 & 0 & -1 & 0 \\
0 & 1 & 0 & -1 \\
0 & 0 & 1 & 0 \\
0 & 0 & 0 & 1
\end{array}\right)+\left(\begin{array}{cccc}
0 & 0 & -1 & 0 \\
0 & 0 & 0 & -1 \\
1 & 0 & 0 & 0 \\
0 & 1 & 0 & 0
\end{array}\right)\left(\begin{array}{cccc}
0 & -1 & 0 & 1 \\
0 & 0 & -1 & 0 \\
0 & 0 & 0 & -1 \\
0 & 0 & 0 & 0
\end{array}\right) \\
& =\left(\begin{array}{cccc}
0 & 1 & 0 & -1 \\
1 & 0 & 0 & 0 \\
0 & 0 & 0 & 1 \\
-1 & 0 & 1 & 0
\end{array}\right) .
\end{aligned}
$$

Example 5.2. $\mathrm{H}$ is a $5 \times 5$ Hankel matrix

$$
H=\left(\begin{array}{lllll}
1 & 1 & 0 & 1 & 1 \\
1 & 0 & 1 & 1 & 0 \\
0 & 1 & 1 & 0 & 0 \\
1 & 1 & 0 & 0 & 1 \\
1 & 0 & 0 & 1 & 0
\end{array}\right)
$$

It is obvious that $\mathrm{H}$ is invertible. By Algorithm 4.2, we have

Step 1. Compute $\hat{v}$ by Lemma 2.2:

$$
\hat{v}=\left(\begin{array}{lllll}
0 & 1 & 1 & 1 & 1
\end{array}\right)^{\top} .
$$

Step 2. Compute $u, z$ by the systems of equations $\mathrm{Hu}=\hat{v}, \mathrm{~Hz}=\mathrm{e}_{1}$ :

$$
u=\left(\begin{array}{c}
2 \\
1 \\
0 \\
-1 \\
-2
\end{array}\right), \quad z=\left(\begin{array}{c}
-1 \\
0 \\
0 \\
1 \\
1
\end{array}\right)
$$

Step 3. Compute $\mathrm{H}^{-1}$ by using the equation (2.3):

$$
\begin{aligned}
\mathrm{H}^{-1}= & \left(\begin{array}{ccccc}
-1 & 0 & 0 & 1 & 1 \\
0 & 0 & 1 & 1 & 1 \\
0 & 1 & 1 & 1 & 0 \\
1 & 1 & 1 & 0 & 0 \\
1 & 1 & 0 & 0 & -1
\end{array}\right)\left(\begin{array}{ccccc}
1 & -2 & -1 & 0 & 1 \\
0 & 1 & -2 & -1 & 0 \\
0 & 0 & 1 & -2 & -1 \\
0 & 0 & 0 & 1 & -2 \\
0 & 0 & 0 & 0 & 1
\end{array}\right) \\
& +\left(\begin{array}{ccccc}
2 & 1 & 0 & -1 & -2 \\
1 & 0 & -1 & -2 & -2 \\
0 & -1 & -2 & -2 & -1 \\
-1 & -2 & -2 & -1 & 0 \\
-2 & -2 & -1 & 0 & 1
\end{array}\right)\left(\begin{array}{ccccc}
0 & -1 & 0 & 0 & 1 \\
0 & 0 & -1 & 0 & 0 \\
0 & 0 & 0 & -1 & 0 \\
0 & 0 & 0 & 0 & -1 \\
0 & 0 & 0 & 0 & 0
\end{array}\right) \\
= & \left(\begin{array}{ccccc}
-1 & 0 & 0 & 1 & 1 \\
0 & -1 & 1 & 0 & 1 \\
0 & 1 & 0 & 0 & -1 \\
1 & 0 & 0 & -1 & 0 \\
1 & 1 & -1 & 0 & -2
\end{array}\right) .
\end{aligned}
$$




\section{The value of formulae}

Let $T=\left[t_{j-k}\right]_{j, k=0}^{n-1}$ be a real symmetric positive-definite Toeplitz matrix of order $n$. There are several well-known $\mathrm{O}\left(\mathrm{n}^{2}\right)$ algorithms for solving the linear system of equations $\mathrm{T} x=\mathrm{b}$ and more recently, several $\mathrm{O}\left(\operatorname{nlog}^{2} n\right)$ algorithms have been developed. See, for example, [1-3, 12, 31] and the references contained therein.

We now propose how the analogue of formulae (2.1) and (2.3) lead to a more efficient way to calculate $\mathrm{T}^{-1} \mathrm{~b}$ and $\mathrm{H}^{-1} \mathrm{~b}$.

It is really worth mentioning that, for real signals filtering, the computational cost of a skew-cyclic convolution is about half of cyclic convolution [5]. That is a strong argument for our algorithm.

According to computational implications of Ammar and Gader [1], we know that the computation of $x=T^{-1} b$, by using our formula (2.1) requires at most $8 \tau(n)+O(n)$ computations, as well as the computation of $x=\mathrm{H}^{-1}$ b using our formula (2.3) requires at most $8 \tau(n)+O(n)$ computations, too.

We list the number of real arithmetic operations (additions and multiplications) required by the Levinson-Durbin, the split Levinson, the generalized Schur, the Gohberg-Semencul and the circulant Gohberg-Semencul algorithms in [1] algorithms for $\mathrm{T}^{-1} \mathrm{~b}$ in Table 1, as well as operation counts for the implementations of formulae (2.1) and (2.3) described above.

The value of our formulae (2.1) and (2.3) increase dramatically in situations in which $T^{-1} b_{k}$ and $\mathrm{H}^{-1} \mathrm{~b}_{\mathrm{k}}$ are to be obtained for several different vectors $b_{k}$. Instances of this situation are in the iterative improvement of solutions.

Table 1: Operation counts.

\begin{tabular}{l||c}
\hline \multicolumn{1}{c|}{ Algorithm } & Number of real arithmetic operations \\
\hline \hline 1. The Levinson-Durbin algorithm for T & $2 n^{2}[1]$ \\
2. The split Levinson algorithm for T & $\frac{3}{2} n^{2}[1]$ \\
3. The generalized Schur algorithm for T & $8 \log _{2}^{2} n-2 n \log _{2} n[1]$ \\
4. The Gohberg-Semencul formula for T & $28 n \log _{2} n[1]$ \\
5. The circulant Gohberg-Semencul formula for T & $18 n \log _{2} n[1]$ \\
\hline 6. The skew circulant GS formula (2.1) for T & $16 n \log _{2} n$ \\
7. The skew left circulant GS formula (2.3) for H & $16 \log _{2} n$ \\
\hline
\end{tabular}

\section{Acknowledgment}

The authors cordially thank the referees for their careful reading and helpful comments. This work were supported by the GRRC program of Gyeonggi Province (GRRC SUWON 2016-B4) and the Natural Science Foundation of China under Grant No. 11271175. Their support is gratefully acknowledged.

\section{References}

[1] G. Ammar, P. Gader, A variant of the Gohberg-Semencul formula involving circulant matrices, SIAM J. Matrix Anal. Appl., 12 (1991), 534-540. 1, 6, 1

[2] G. S. Ammar, W. B. Gragg, The generalized Schur algorithm for the superfast solution of Toeplitz systems, Rational approximation and applications in mathematics and physics, Łańcut, (1985), 315-330, Lecture Notes in Math., Springer, Berlin, (1987).

[3] G. S. Ammar, W. B. Gragg, Superfast solution of real positive definite Toeplitz systems, SIAM Conference on Linear Algebra in Signals, Systems, and Control, Boston, Mass., (1986), SIAM J. Matrix Anal. Appl., 9 (1988), 61-76. 6

[4] A. Ben-Artzi, T. Shalom, On inversion of Toeplitz and close to Toeplitz matrices, Linear Algebra Appl., 75 (1986), 173-192. 1

[5] A. Daher, E. H. Baghious, G. Burel, Fast algorithm for optimal design of block digital filters based on circulant matrices, IEEE Signal Process. Lett., 15 (2008), 637-640. 6

[6] M. I. Español, M. E. Kilmer, Multilevel approach for signal restoration problems with Toeplitz matrices, SIAM J. Sci. Comput., 32 (2010), 299-319. 1 
[7] D.-Q. Fu, Z.-L. Jiang, Y.-F. Cui, S. T. Jhang, New fast algorithm for optimal design of block digital filters by skew-cyclic convolution, IET Signal Process., 8 (2014), 633-638. 1

[8] I. Gohberg, N. Krupnik, A formula for the inversion of the finite Toeolitz matrices, (Russian) Mat. Issled., 7 (1972), 272-283. 1

[9] I. Gohberg, A. Semencul, On the inversion of finite Toeplitz matrices and their continuous analogs, (Russian) Mat. issled., 2 (1972), 201-233. 1

[10] G. Heinig, On the reconstruction of Toeplitz matrix inverses from columns, Linear Algebra Appl., 350 (2002), $199-212$. 1

[11] G. Heinig, K. Rost, Algebraic methods for Toeplitz-like matrices and operators, Mathematical Research, AkademieVerlag, Berlin, (1984). 1

[12] J. R. Jain, An efficient algorithm for a large Toeplitz set of linear equations, IEEE Trans. Acoust. Speech Signal Process., 27 (1979), 612-615. 6

[13] Z.-L. Jiang, J.-X. Chen, The explicit inverse of nonsingular conjugate-Toeplitz and conjugate-Hankel matrices, J. Appl. Math. Comput., 53 (2015), 1-16. 1

[14] X.-Y. Jiang, K.-C. Hong, Explicit inverse matrices of Tribonacci skew circulant type matrices, Appl. Math. Comput., 268 (2015), 93-102. 2.3, 2.4

[15] Z.-L. Jiang, T.-Y. Tam, Y.-F. Wang, Inversion of conjugate-Toeplitz matrices and conjugate-Hankel matrices, Linear Multilinear Algebra, 65 (2016), 256-268. 1

[16] Z.-L. Jiang, D.-D. Wang, Explicit group inverse of an innovative patterned matrix, Appl. Math. Comput., 274 (2016), 220-228. 1

[17] Z.-L. Jiang, Z.-X. Zhou, Circulant matrices, Chengdu Technology University Publishing Company, Chengdu, (1999). $2.3,2.4$

[18] G. Labahn, T. Shalom, Inversion of Toeplitz matrices with only two standard equations, Linear Algebra Appl., 175 (1992), 143-158. 1

[19] G. Labahn, T. Shalom, Inversion of Toeplitz structured matrices using only standard equations, Linear Algebra Appl., 207 (1994), 49-70. 1

[20] L. Lerer, M. Tismenetsky, Generalized Bezoutian and the inversion problem for block matrices, I, General scheme, Integral Equations Operator Theory, 9 (1986), 790-819. 1

[21] X.-G. Lv, T.-Z. Huang, A note on inversion of Toeplitz matrices, Appl. Math. Lett., 20 (2007), 1189-1193. 1

[22] M. Naffouti, A. Baccari, Characterization of a class of copositive matrices, Commun. Optim. Theory, 2016 (2016), 7 pages. 1

[23] M. J. Narasimha, Linear convolution using skew-cyclic convolutions, IEEE Signal Process. Lett., 14 (2007), 173-176. 1

[24] M. K. Ng, Iterative methods for Toeplitz systems, Numerical Mathematics and Scientific Computation, Oxford University Press, New York, (2004). 1

[25] M. K. Ng, J.-Y. Pan, Weighted Toeplitz regularized least squares computation for image restoration, SIAM J. Sci. Comput., 36 (2014), B94-B121. 1

[26] M. K. Ng, K. Rost, Y.-W. Wen, On inversion of Toeplitz matrices, Linear Algebra Appl., 348 (2002), 145-151. 1

[27] W. F. Trench, An algorithm for the inversion of finite Toeplitz matrices, J. Soc. Indust. Appl. Math., 12 (1964), 515-522. 1

[28] Y.-W. Wen, M. K. Ng, W.-K. Ching, H. liu, A note on the stability of Toeplitz matrix inversion formulas, Appl. Math. Lett., 17 (2004), 903-907. 1

[29] Y.-P. Zheng, S.-G. Shon, Exact determinants and inverses of generalized Lucas skew circulant type matrices, Appl. Math. Comput., 270 (2015), 105-113. 2.3, 2.4

[30] S. Zohar, Toeplitz matrix inversion: The algoritm of W. F. Trench, J. Assoc. Comput. Mach., 16 (1969), 592-601. 1

[31] S. Zohar, The solution of a Toeplitz set of linear equations, J. Assoc. Comput. Mach., 21 (1974), 272-276. 3, 6 